\title{
Ausbeutesteigerung mittels Membranreaktoren bei Parallel- und Folgereaktionen
}

\author{
Sascha Thomas, Frank Klose, Andreas Seidel-Morgenstern*
}

Membranreaktoren besitzen das Potential zur Steigerung der Selektivität der Bildung eines gewünschten Reaktionsproduktes durch örtlich verteilte Eduktzugabe. In dieser Arbeit wurde die optimale Dosierung mit dem Ziel der Maximierung der Ausbeute eines Zielproduktes sowohl theoretisch als auch experimentell untersucht. Als Modellreaktion wurde die oxidative Dehydrierung von Ethan zu Ethylen unter Verwendung eines $\mathrm{VO}_{\mathrm{x}} / \gamma-\mathrm{Al}_{2} \mathrm{O}_{3}$-Katalysators gewählt.

\section{Einleitung}

Trotz der in den letzten Jahren auf dem Gebiet der Katalysatorforschung erreichten Fortschritte [u.a. 1,2] besteht bei vielen technisch relevanten Reaktionen ein erheblicher Bedarf an Methoden zur Verbesserung der Ausbeute an gewünschten Produkten. Typische Beispiele stellen Partialoxidationen dar, bei denen in der Regel die Produktselektivität durch die Bildung von unerwünschten Totaloxidationsprodukten limitiert ist [u.a. 3,4].

Beim Auftreten von unerwünschten Folgereaktionen führt die Vermeidung von Rückvermischungen zu einer Selektivitätssteigerung des gewünschten Zwischenproduktes. Neben der einfacheren Kontrollierbarkeit ist dies einer der Hauptgründe, warum Oxidationsreaktionen bevorzugt in Rohrreaktoren durchgeführt werden [u.a. 5]. Typischerweise werden in konventionellen Festbettreaktoren (FBR) alle Edukte am Reaktoreintritt gemeinsam dem Katalysatorbett zugeführt. In diesem Fall können bei Vorgabe der Verweilzeit die lokalen Reaktionsgeschwindigkeiten und damit die am Reaktoraustritt resultierenden Produktselektivitäten bzw. -ausbeuten lediglich durch die Variation der Temperatur beeinflusst und gesteuert werden. Deshalb wurden zur Einstellung maximaler

\footnotetext{
* Dr.-Ing. S. Thomas, Fraunhofer IFF, Sandtorstr. 22, 39106 Magdeburg, Germany; Dr. F. Klose, Otto-vonGuericke-Universität Magdeburg, IFAT, Universitätsplatz 2, 39104 Magdeburg, Germany und Max-PlanckInstitut für Dynamik komplexer technischer Systeme, Sandtorstr. 1, 39106 Magdeburg, Germany; Prof. Dr.-Ing. habil. A. Seidel-Morgenstern (Korrespondenzautor, anseidel@vst.uni-magdeburg.de), Otto-von-GuerickeUniversität Magdeburg, IVT, Universitätsplatz 2, 39104 Magdeburg, Germany und Max-Planck-Institut für Dynamik komplexer technischer Systeme, Sandtorstr. 1, 39106 Magdeburg, Germany.
} 
Produktausbeuten Konzepte zur Ermittlung optimaler axialer Temperaturprofile vorgeschlagen [u.a. 6]. Alternativ dazu wurde die Verbesserung von Zwischenproduktselektivitäten bzw. -ausbeuten durch eine Verteilung von Katalysatoren unterschiedlicher Aktivität entlang dem Reaktionsweg untersucht [7].

Neben der Abhängigkeit von der Temperatur und den Katalysatoreigenschaften ist bekannt, dass in Parallel- und Folgereaktionen die Zwischenproduktselektivitäten und -ausbeuten durch eine Veränderung der lokalen Eduktkonzentrationen beeinflusst werden können [u.a. 8]. Durch die Zuführung eines Eduktes am Eintritt eines Rohrreaktors und die verteilte Zugabe eines anderen Eduktes entlang der axialen Reaktorkoordinate können die lokalen Konzentrationsprofile gezielt eingestellt werden. Aus diesem Grund bietet die örtlich verteilte Zuführung der Edukte eine weitere Möglichkeit der Erhöhung der Ausbeute an einem gewünschten Produkt. Eine grundlegende Voraussetzung für eine gezielte Anwendung dieses Konzeptes ist offensichtlich die Kenntnis der Kinetik der ablaufenden Reaktionen. Dabei ist bekannt, dass insbesondere die Reaktionsordnungen der verteilt zugeführten Komponente von entscheidender Bedeutung sind $[9,10]$.

Eine einfache und attraktive Möglichkeit für eine verteilte Zugabe mindestens eines Eduktes bieten Membranreaktoren (MR). Dabei bilden durchlässige rohrförmige Membranen die Reaktorwand [11]. Vor dem geschilderten Hintergrund ist es Ziel dieser Arbeit, die verteilte Eduktzugabe sowohl theoretisch als auch experimentell $\mathrm{zu}$ untersuchen und einen Membranreaktor mit einem konventionellen Festbettreaktor zu vergleichen [12].

\section{Theoretische Vorbetrachtungen}

In den theoretischen Vorbetrachtungen zur Bewertung des Potentials einer optimalen Eduktverteilung sollen zum einen eine Folgereaktion sowie eine Parallelreaktion betrachtet werden. In Fall I reagieren zwei Edukte, E1 und E2, zum gewünschten Produkt GP, das in der unerwünschten Folgereaktion mit dem Edukt E2 zum unerwünschten Endprodukt UP weiter umgesetzt werden kann. Das Reaktionsschema für Fall I lautet:

$$
\begin{aligned}
& E 1+E 2 \ddagger G P \\
& G P+E 2 \ddagger U P
\end{aligned}
$$


Im zweiten Fall sollen zwei Edukte, E1 und E2, sowohl in der gewünschten Reaktion zum Zielprodukt $G P$ als auch in der unerwünschten Parallelreaktion zum Nebenprodukt $U P$ reagieren können. Fall II kann wie folgt beschrieben werden:

$E 1+E 2 \ddagger G P$

$E 1+E 2 \ddagger U P$

In beiden betrachteten Reaktionssystemen soll im Folgenden das Edukt E1 die „wertvollere“, möglichst weitgehend umzusetzende Eduktkomponente darstellen und das Edukt E2 die optimal zu dosierende Komponente bezeichnen.

\subsection{Maximierung der Ausbeute durch Eduktverteilung}

Die am Austritt eines Rohrreaktors sich einstellende Ausbeute hinsichtlich eines gewünschten Produktes, $A_{G P}$, stellt eine integrale Größe dar und ergibt sich aus dem Produkt der integralen Selektivität zu diesem Produkt, $S_{G P}^{\text {int }}$, mit dem Umsatz des Eduktes $E 1, U_{E 1}$ :

$$
A_{G P}=S_{G P}^{i n t} \cdot U_{E 1}
$$

Eine andere Möglichkeit zur Berechnung der Ausbeute bietet die Verwendung der differentiellen Selektivität, $S_{G P}^{\text {diff }}$, wobei in diesem Fall das folgende Integral entlang dem Reaktionsweg bis zum Umsatz am Reaktoraustritt, $U_{E 1}^{\text {end }}$, gelöst werden muss:

$$
A_{G P}=\int_{0}^{U_{E I}^{\text {end }}} S_{G P}^{\text {diff }} \cdot d U_{E 1}
$$

Die differentielle Selektivität entspricht dabei dem Verhältnis der Bruttobildungsgeschwindigkeit der betrachteten Produktkomponente zur Verbrauchsgeschwindigkeit der entsprechenden Eduktkomponente [13] und kann allgemein als Funktion der lokalen Geschwindigkeiten von $\mathrm{M}$ ablaufenden Reaktionen wie folgt ausgedrückt werden:

$$
S_{G P}^{\text {diff }}=\sum_{j=1}^{M} v_{G P, j} \cdot r_{j} / \sum_{j=1}^{M}\left|v_{E 1, j}\right| \cdot r_{j}
$$


Ziel ist es nun, mit Hilfe einer optimierten Dosierung des Eduktes E2 ein optimales Konzentrationsprofil im Reaktionsraum einzustellen, um in Gleichung 6 das Integral zu maximieren. Für isobare Verhältnisse lautet dann die Optimierungsaufgabe, die Ableitung von Gleichung 6 nach dem Molanteil der zu dosierenden Eduktkomponente Null zu setzen:

$$
\frac{\partial A_{G P}}{\partial x_{E 2}}=\frac{\partial}{\partial x_{E 2}}\left(\int_{0}^{U_{E 1}^{e n d}} S_{G P}^{d i f f} \cdot d U_{E 1}\right)=0
$$

Unter Nutzung der Leibnitz'schen Integrationsbeziehung [14]

$$
\frac{\partial}{\partial x} \int_{a(x)}^{b(x)} f(x, z) \cdot d z=\int_{a(x)}^{b(x)} \frac{\partial f(x, z)}{\partial x} \cdot d z+f(b(x), x) \cdot \frac{\partial b}{\partial x}-f(a(x), x) \cdot \frac{\partial a}{\partial x}
$$

kann Gleichung 8 mit $f(x, z)=S_{G P}^{\text {diff }}\left(x_{E 2}, U_{E 1}\right)$ auch wie folgt formuliert werden:

$$
\frac{\partial A_{G P}}{\partial x_{E 2}}=\int_{0}^{U_{E 1}^{\text {end }}} \frac{\partial S_{G P}^{\text {diff }}}{\partial x_{E 2}} \cdot d U_{E 1}+S_{G P}^{\text {diff }}\left(x_{E 2}, U_{E 1}^{\text {end }}\right) \cdot \frac{\partial U_{E 1}^{\text {end }}}{\partial x_{E 2}}=0
$$

Für eine weitere Vereinfachung der Gleichung 10 und deren Lösung sollen im Folgenden die Folge- und die Parallelreaktion getrennt voneinander betrachtet werden.

\section{$\underline{\text { Fall I (Folgereaktion) }}$}

Im Fall von Folgereaktionen gilt, dass eine maximale Ausbeute an der Reaktorposition vorliegt, an der der Betrag der gewünschten Reaktionsgeschwindigkeit, $r_{G R}$, dem der unerwünschten Reaktion, $r_{U R}$, entspricht. Der Zähler von Gleichung 7 und damit die differentielle Selektivität sind an dieser Position Null. Bei einer Weiterführung der Reaktion wird auf Grund der höheren Reaktionsgeschwindigkeit der unerwünschten Folgereaktion die differentielle Selektivität immer negative Zahlenwerte besitzen und durch die Folgereaktion mehr Zwischenprodukt verbraucht als in der gewünschten Reaktion entsteht. An der Stelle des Ausbeutemaximums gilt demnach folgende Bedingung für die differentielle Selektivität zum gewünschten Zwischenprodukt:

$$
S_{G P}^{\text {diff }}\left(x_{E 2}, U_{E 1}^{\text {end }}\right)=\frac{r_{G R}-r_{U R}}{r_{G R}}=0
$$


Durch Einsetzen von Gleichung 11 vereinfacht sich Gleichung $10 \mathrm{zu}$ :

$$
\frac{\partial A_{G P}}{\partial x_{E 2}}=\int_{0}^{U_{E 1}^{e n d}} \frac{\partial S_{G P}^{\text {diff }}}{\partial x_{E 2}} \cdot d U_{E 1}=0
$$

Zur Erfüllung von Gleichung 12 muss die Ableitung von $S_{G P}^{\text {diff }}$ nach $x_{E 2}$ an allen Reaktorpositionen gleich Null sein:

$$
\frac{\partial S_{G P}^{\text {diff }}}{\partial x_{E 2}}=0
$$

Die Lösung der Gleichung 13 erlaubt die Ermittlung eines optimalen „,lokalen“ Molanteils der dosierten Komponente E2 zur Maximierung der Ausbeute des gewünschten Zwischenproduktes. Eine Realisierung des Dosierkonzeptes erfordert die Einstellung dieses optimalen Molanteils durch eine Zuführung dieser Komponente über die Wand.

\section{$\underline{\text { Fall II (Parallelreaktion) }}$}

Bei einer Parallelreaktion ist die maximale Ausbeute hinsichtlich des gewünschten Produktes erreicht, wenn das umzusetzende Edukt $E 1$ vollständig umgesetzt ist $\left(U_{E 1}^{\text {end }}=100 \%\right)$. In diesem Fall sind an der Position des Ausbeutemaximums die Geschwindigkeit der gewünschten Reaktion und damit die differentielle Selektivität gleich Null:

$$
S_{G P}^{\text {diff }}\left(x_{E 2}, U_{E 1}^{\text {end }}=100 \%\right)=0
$$

Somit reduziert sich Gleichung 10 auch für eine Parallelreaktion auf Gleichung 12. Ebenfalls gilt damit für eine Parallelreaktion, dass zur Maximierung der Zielproduktausbeute die Ableitung der differentiellen Selektivität hinsichtlich des gewünschten Produktes nach dem Molanteil der dosierten Komponente an jeder Reaktorposition gleich Null sein muss (Gleichung 13).

Durch die Anwendung von 3 unterschiedlichen mathematischen Methoden konnte in einer umfangreichen Simulationsstudie für verschiedene Reaktionssysteme wie Parallelreaktion mit einem und zwei Edukten, Folgereaktion mit zwei Edukten sowie Parallel- und Folgereaktion mit zwei Edukten unter Einbeziehung von analytischen Lösungen [15, 16] sowie numerischer Optimierung unter Verwendung des SQP-Algorithmus [17] die Möglichkeiten der Steigerung 
der Ausbeute hinsichtlich der Zielkomponente durch die optimale Dosierung eines Eduktes entlang der Reaktorachse demonstriert werden. Des Weiteren konnten grundlegende Zusammenhänge zwischen den Betriebsgrößen (Verweilzeit sowie Eduktmolanteil) und den Zielgrößen (differentielle und integrale Selektivität, Umsatz sowie Ausbeute) herausgearbeitet werden [12]. Im Folgenden soll exemplarisch die Möglichkeit der Steigerung der Ausbeute an einem gewünschten Produkt durch eine optimale Eduktdosierung entlang dem Reaktionsweg für zwei konkrete Beispiele demonstriert werden.

\subsection{Maximale Ausbeute im Fall einer Folgereaktion (Fall I)}

Die differentielle Selektivität hinsichtlich des gewünschten Zwischenproduktes GP lässt sich für eine einfache Folgereaktion in Abhängigkeit von den Reaktionsgeschwindigkeiten wie folgt ausdrücken:

$S_{G P}^{\text {diff }}=\frac{r_{G R}-r_{U R}}{r_{G R}}=1-\frac{1}{r_{G R} / r_{U R}}$

Es ist $\mathrm{zu}$ erkennen, dass am Maximum des Verhältnisses der gewünschten Reaktionsgeschwindigkeit zur Geschwindigkeit der unerwünschten Folgereaktion, $r_{G R} / r_{U R}$, auch ein Maximum der differentiellen Selektivität, $S_{G P}^{\text {diff }}$, auftreten muss. Die Bedingung zur Maximierung der Ausbeute kann demzufolge in Analogie zu Gleichung 13 auch für das Verhältnis der Reaktionsgeschwindigkeiten formuliert werden:

$$
\frac{\partial\left(r_{G R} / r_{U R}\right)}{\partial x_{E 2}}=0
$$

Für das Reaktionsschema der Folgereaktionen (Gleichungen 1 und 2) werden exemplarisch die folgenden einfachen hypothetischen reaktionskinetischen Ansätze 17 und 18 für die gewünschte und unerwünschte Reaktion angenommen:

$$
\begin{aligned}
& r_{G R}=k_{G R} \cdot x_{E 1} \cdot \frac{x_{E 2}^{2}}{\alpha \cdot x_{E 2}^{2}+1} \\
& r_{U R}=k_{U R} \cdot x_{G P} \cdot x_{E 2}
\end{aligned}
$$


Damit ergibt sich folgendes Verhältnis der Geschwindigkeiten der gewünschten zur unerwünschten Reaktion in Abhängigkeit vom Molanteil der Edukte:

$$
\frac{r_{G R}}{r_{U R}}=\frac{k_{G R}}{k_{U R}} \cdot \frac{x_{E 1}}{x_{G P}} \cdot \frac{x_{E 2}}{\alpha \cdot x_{E 2}^{2}+1}
$$

Gleichung 19 verdeutlicht, dass eine Erhöhung des Molanteils von E1 zu einer Steigerung des Verhältnisses der Reaktionsgeschwindigkeiten und damit der differentiellen Selektivität führt. Die Eduktkomponente E1 sollte demnach nicht verteilt, sondern am Reaktoreintritt mit maximaler Konzentration der Reaktionszone zugeführt werden. Dagegen ist zu erkennen, dass der Einfluss der in beiden Reaktionen auftretenden Komponente E2 von der Größe des Parameters $\alpha$ abhängt. Mit Hilfe der Gleichungen 16 und 19 ergibt sich der optimale Molanteil von E2 zur Maximierung des Verhältnisses $r_{G R} / r_{U R}$ in Abhängigkeit von $\alpha$ wie folgt:

$x_{E 2}^{o p t}=\frac{1}{\sqrt{\alpha}}$

In Abb. 1 sind die Molanteilverläufe der Komponenten E1, E2 und GP in Abhängigkeit von einer dimensionslosen axialen Reaktorlängenkoordinate $\xi$ für einen rohrförmigen und isotherm betriebenen Plug-Flow-Reaktor mit Einleitung der Edukte E1 und E2 am Reaktoreintritt und zusätzlicher optimaler Dosierung des Eduktes E2 entlang des Reaktors im Fall von $\alpha=100$ dargestellt. Zur Gewährleistung einer maximalen Produktion des Zielproduktes ab dem Reaktoreintritt muss das Edukt E2 mit dem optimalen Molanteil von $x_{E 2}^{o p t}=0.1$ (Gleichung 20) zugeleitet werden. Des Weiteren muss zur Aufrechterhaltung des optimalen Molanteils von E2 der dosierte Molenstrom der Komponente E2 der in den Reaktionen $r_{G R}$ und $r_{U R}$ verbrauchten Menge an E2 entsprechen. Unter Berücksichtigung eines konstanten Molanteils des Eduktes E2 im Reaktionsraum $\left(x_{E 2}=\mathrm{const}=x_{E 2}^{o p t}\right)$ liefert die Komponentenbilanz von E2 die optimal zu dosierende Menge des Eduktes E2 als Funktion der Reaktorposition:

$$
j_{E 2}^{o p t}(\xi)=\frac{D_{R o h r}}{4} \cdot\left[r_{G R}(\xi)+r_{U R}(\xi)\right]
$$


Abbildung 1.

a) Molanteilverläufe, b) Differenz zwischen gewünschter und unerwünschter Reaktionsgechwindigkeit $r_{G R}-r_{U R}$, c) Umsatz $U_{E l}$, differentielle sowie integrale Selektivität $S_{E 2}^{\text {diff }}$ bzw. $S_{E 2}^{\text {int }}$ und d) Molenstromdichte des dosierten Eduktes E2 in Abhängigkeit von der dimensionslosen Reaktorlängenkoordinate $\xi$ für $\alpha=100, k_{G R}=6 \cdot k_{U R}=3000 \mathrm{~mol} /\left(\mathrm{s} \mathrm{m}^{3}\right), \alpha_{e s}(\xi=0)=1 \mathrm{~mol} / \mathrm{s}, F=0,05$ $\mathrm{m}^{2}, x_{E 1}(\xi=0)=0,6, x_{E 2}(\xi=0)=x_{E 2}^{o p t}=0,1$ und $\tau=4 \mathrm{~s}$.
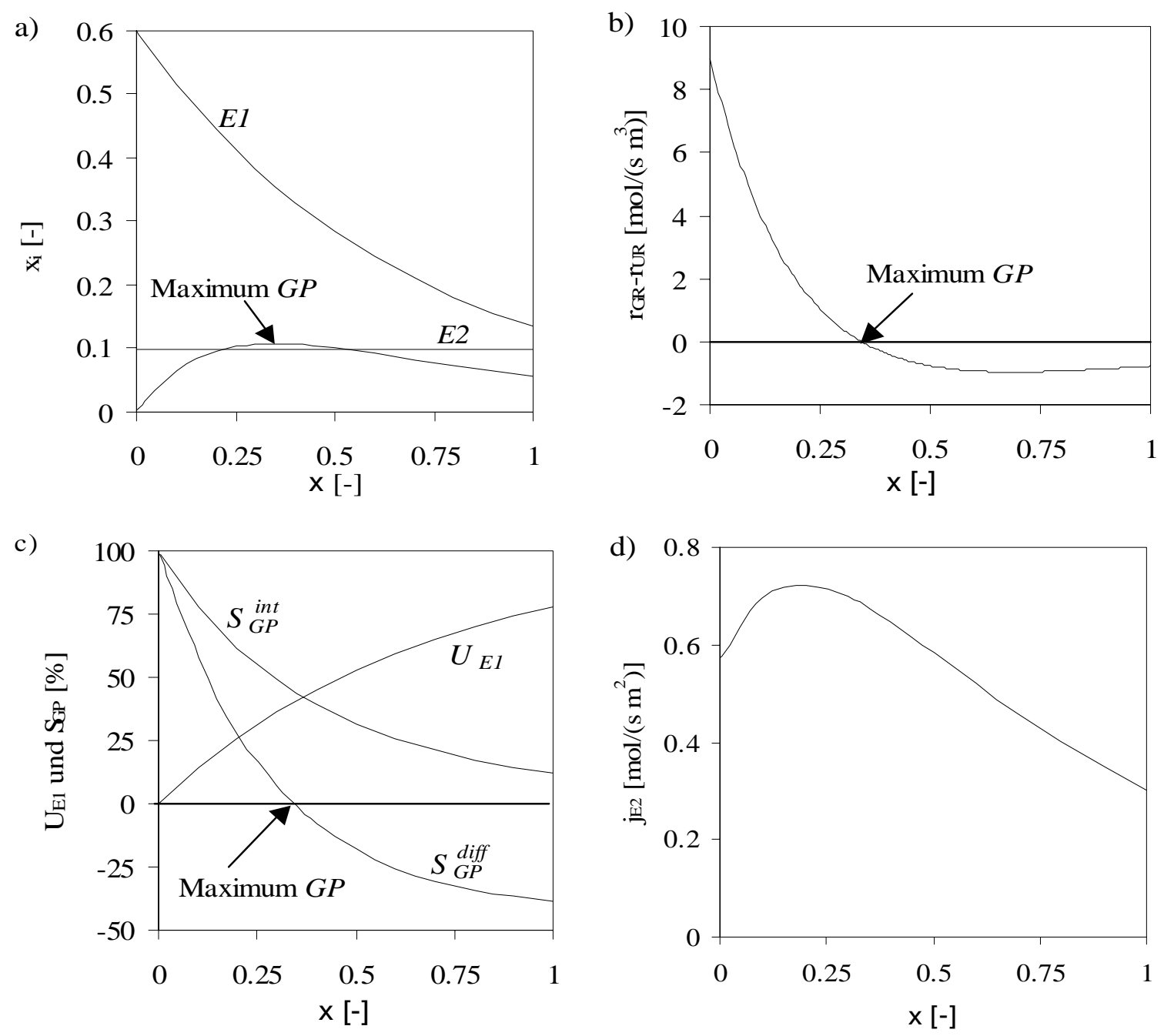

In Abb. 1a ist zu sehen, dass auf Grund der Abreaktion der Molanteil der Komponente E1 zum Reaktoraustritt abnimmt. Da die Komponente E2 am Eintritt und entlang des Reaktors optimal zudosiert wird, liegt in der gesamten Reaktionszone der optimale Molanteil von $x_{E 2}^{o p t}=0.1$ vor. Die differentielle Selektivität hinsichtlich des gewünschten Produktes ist somit an allen Reaktorpositionen maximal. Der Molanteil von $G P$ steigt zunächst an, erreicht sein Maximum und fällt danach ab. An der Position des Maximums entspricht der Betrag der gewünschten Reaktionsgeschwindigkeit dem der unerwünschten Reaktionsgeschwindigkeit (Abb. 1b). Nach diesem Punkt wird mehr Zwischenprodukt verbraucht, als in der gewünschten Reaktion entstehen kann. 
Wie in Abb. 1c zu erkennen ist, beträgt die differentielle Selektivität zum gewünschten Produkt $G P$ an der Stelle des Molanteilmaximums Null. Danach wird $S_{E 2}^{\text {diff }}$ negativ. Da die Geschwindigkeit der Folgereaktion auf Grund des wachsenden Molanteils des Zwischenproduktes mit zunehmender Länge steigt, sinkt die differentielle und integrale Selektivität zum Reaktoraustritt ab. An der Position des Zielproduktmaximums muss die Reaktion zur Erzielung der optimalen Produktausbeute abgebrochen werden. Somit muss bei vorgegebener Reaktorlänge die Verweilzeit bzw. bei fixer Verweilzeit die Reaktorlänge zur Erzielung des Ausbeutemaximums am Reaktoraustritt angepasst werden. Die optimal zu dosierende Molenstromdichte, die zur Gewährleistung des konstanten Molanteils des Eduktes E2 notwendig ist und mit Gleichung 21 berechnet wurde, ist in Abb. 1d dargestellt.

\subsection{Maximale Ausbeute im Fall einer Parallelreaktion (Fall II)}

Für die differentielle Selektivität hinsichtlich des gewünschten Produktes im Fall einer einfachen Parallelreaktion gilt:

$$
S_{G P}^{\text {diff }}=\frac{r_{G R}}{r_{G R}+r_{U R}}=\frac{1}{1+\frac{1}{r_{G R} / r_{U R}}}
$$

Analog zur Folgereaktion ist auch im Fall der Parallelreaktion zu erkennen, dass die Maximierung des Verhältnisses von gewünschter Reaktionsgeschwindigkeit zur Geschwindigkeit der unerwünschten Reaktion zu einer maximalen differentiellen Selektivität führt. Daraus folgt wiederum, dass ein optimaler Molanteil der dosierten Komponente E2 auch bei einer Parallelreaktion durch die Ableitung von $r_{G R} / r_{U R}$ nach dem Molanteil dieser Eduktkomponente ermittelt werden kann (Gleichung 16).

In dem nachfolgenden Beispiel soll basierend auf dem angenommenen Reaktionsschema der Parallelreaktion (Gleichungen 3 und 4) der reaktionskinetische Ansatz nach Gleichung 17 für die gewünschte Reaktion gelten. Die Gleichung zur Beschreibung der Geschwindigkeit der unerwünschten Nebenreaktion enthält nun im Gegensatz zur Folgereaktion (Gleichung 18) beide Eduktkomponenten $E 1$ und $E 2$ :

$$
r_{U R}=k_{U R} \cdot x_{E 1} \cdot x_{E 2}
$$


Das Verhältnis von gewünschter zu unerwünschter Reaktionsgeschwindigkeit ergibt sich unter Nutzung der einfachen reaktionskinetischen Ansätzen 17 und 23 in Abhängigkeit vom Molanteil der Edukte wie folgt:

$$
\frac{r_{G R}}{r_{U R}}=\frac{k_{G R}}{k_{U R}} \cdot \frac{x_{E 2}}{\alpha \cdot x_{E 2}^{2}+1}
$$

In Gleichung 24 ist zu erkennen, dass lediglich der Molanteil der Komponente E2 einen Einfluss auf das Verhältnis der Reaktionsgeschwindigkeiten und damit auf die differentielle Selektivität besitzt. Zur Ermittlung des optimalen Molanteils der Komponente E2 wird die Ableitung von Gleichung 24 nach dem Molanteil von E2 gleich Null gesetzt. Auf Grund des im Vergleich zur Folgereaktion unveränderten Einflusses des Molanteils der dosierten Eduktkomponente E2 ergibt sich für die Parallelreaktion die gleiche Beziehung des optimalen Molanteils als Funktion des Parameters $\alpha$ wie für die Folgereaktion (siehe Gleichung 20).

Abbildung 2 enthält die Molanteilverläufe von E1, E2 und des Zielproduktes GP in Abhängigkeit von der dimensionslosen Reaktorlängenkoordinate $\xi$ für einen rohrförmigen und isotherm betriebenen Plug-Flow-Reaktor mit Einleitung der Edukte E1 und E2 am Reaktoreintritt und zusätzlicher optimal verteilter Dosierung des Eduktes E2 für $\alpha=100$. Auch für die Parallelreaktionen folgt gemäß Gleichung 20 ein optimaler Molanteil für E2 von $x_{E 2}^{o p t}=0,1$. In Abb. 2a ist zu erkennen, dass der Molanteil von E1 auf Grund der Abreaktion kontinuierlich sinkt und einen Austrittwert von 0 erreicht. Der Molanteil des Eduktes E2 bleibt infolge der optimalen Zudosierung durch die Membran entlang der Reaktorachse konstant. Der Molanteil des gewünschten Produktes GP steigt kontinuierlich auf den maximalen Endwert an. Auf Grund der vollständigen Abreaktion des Eduktes E1, liegt am Reaktoraustritt ein Umsatz von $100 \%$ vor (Abb. 2b). Da die differentielle Selektivität hinsichtlich des gewünschten Produktes GP lediglich von der Temperatur sowie von dem Molanteil des dosierten Eduktes E2 abhängt und diese Größen im gesamten Reaktionsraum konstant sind, ergibt sich ebenfalls ein konstanter Wert für die differentielle Selektivität hinsichtlich GP (Abb. 2b).

Die Geschwindigkeiten der gewünschten sowie der unerwünschten Reaktion und damit die Molenstromdichte des dosierten Eduktes E2 (Gleichung 21) fallen infolge des sinkenden Molanteils des Eduktes El entlang der Reaktorlängenkoordinate ab und erreichen am Reaktoraustritt den Wert Null (Abb. 2c). 
Abbildung 2.

a) Molanteilverläufe, b) Umsatz $U_{E 1}$ und differentielle Selektivität $S_{E 2}^{\text {diff }}$ sowie c) Molenstromdichte des dosierten Eduktes E2 in Abhängigkeit von der dimensionslosen Reaktorlängenkoordinate $\xi$ für $\alpha=100, k_{G R}=10 \cdot k_{U R}=10^{4} \mathrm{~mol} /\left(\mathrm{s} \mathrm{m}^{3}\right), \underset{g}{\alpha_{g e s}}(\xi=0)=1 \mathrm{~mol} / \mathrm{s}, F=0,01$ $\mathbf{m}^{2}, x_{E 1}(\xi=0)=0,9, x_{E 2}(\xi=0)=x_{E 2}=x_{E 2}^{o p t}=0,1$ und $\tau=1,6 \mathrm{~s}$.
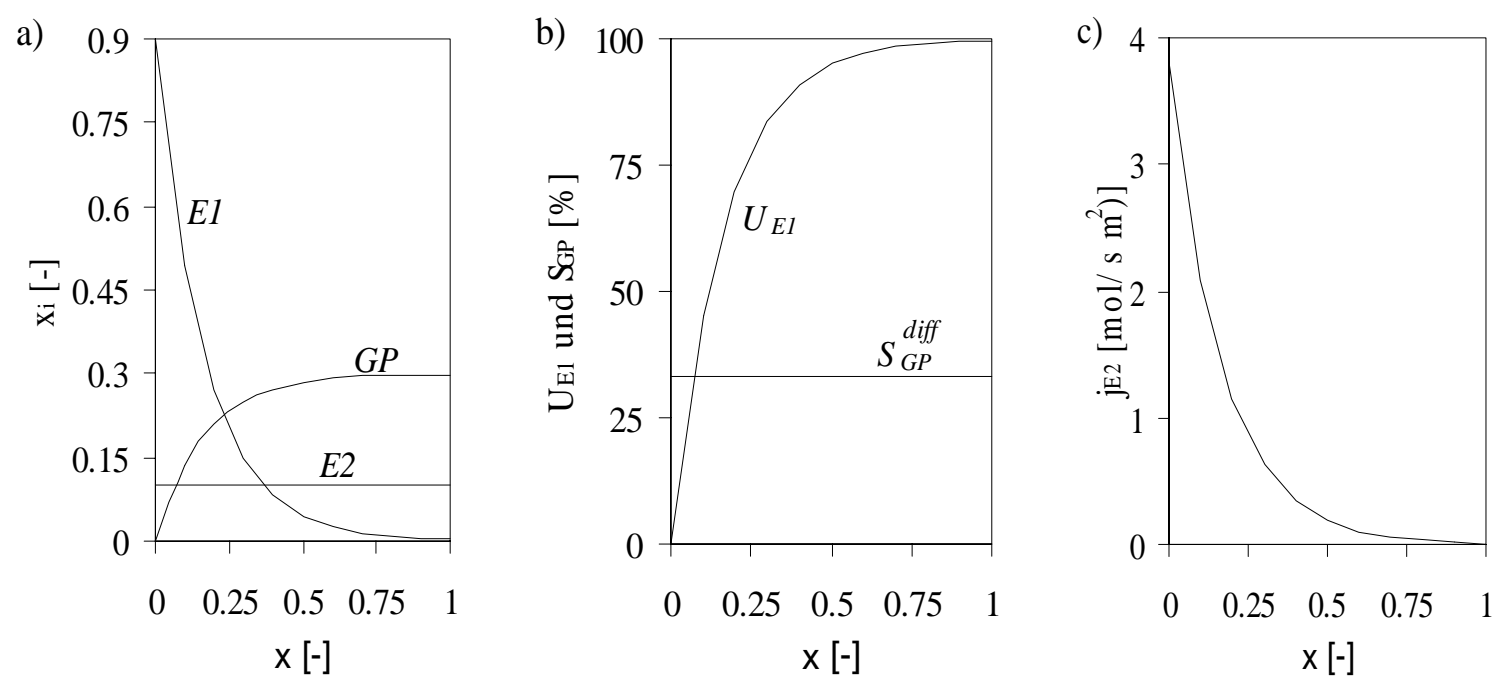

Für beide Fälle I und II, d.h. sowohl für die Folge- als auch für die Parallelreaktionen, ist die optimale Molenstromdichte des dosierten Eduktes E2 eine spezifische Funktion der dimensionslosen Reaktorlängenkoordinate (Abb. 1d und 2c). Die praktische Realisierung eines kontinuierlichen Dosierprofils entlang der Reaktorlänge ist schwierig. Eine Möglichkeit der praktischen Umsetzung eines optimalen Verlaufes der Molenstromdichte entlang der Reaktorlängenkoordinate bietet beispielsweise eine diskrete Eduktzugabe mittels Düsen an bestimmten Reaktorpositionen. Weiterhin ist zur Eduktdosierung eine Reihenschaltung von mehreren MR mit jeweils unterschiedlichen Dosierungsströmen denkbar, wie sie bereits erfolgreich bei der Einstellung von Verweilzeitprofilen getestet werden konnte [18]. Im Folgenden sollen experimentelle Ergebnisse für eine einstufige Dosierung in einem MR vorgestellt und mit denen des konventionellen FBR verglichen werden.

\section{Experimente}

Zur experimentellen Untersuchung des beschriebenen Dosierkonzeptes wurde als Modellreaktion die oxidative Dehydrierung von Ethan zu Ethylen untersucht:

$\mathrm{C}_{2} \mathrm{H}_{6}+0.5 \mathrm{O}_{2} \rightarrow \mathrm{C}_{2} \mathrm{H}_{4}+\mathrm{H}_{2} \mathrm{O}$ 
Neben der gewünschten Reaktion (25) laufen die folgenden unerwünschten Folge- und Parallelreaktionen ab:

$$
\begin{aligned}
& \mathrm{C}_{2} \mathrm{H}_{4}+2 \mathrm{O}_{2} \rightarrow 2 \mathrm{CO}+2 \mathrm{H}_{2} \mathrm{O} \\
& \mathrm{C}_{2} \mathrm{H}_{4}+3 \mathrm{O}_{2} \rightarrow 2 \mathrm{CO}_{2}+2 \mathrm{H}_{2} \mathrm{O} \\
& \mathrm{C}_{2} \mathrm{H}_{6}+3.5 \mathrm{O}_{2} \rightarrow 2 \mathrm{CO}_{2}+3 \mathrm{H}_{2} \mathrm{O}
\end{aligned}
$$

Hierbei wurde ein selbstpräparierter Vanadiumoxid-Katalysator $\left(\gamma-\mathrm{Al}_{2} \mathrm{O}_{3}\right.$-Träger, Vanadiumgehalt 1,37 \%, BET-Oberfläche $167 \mathrm{~m}^{2} / \mathrm{g}$ ) eingesetzt [18]. In ersten reaktionskinetischen Messungen zeigte sich, dass eine Absenkung des Partialdruckes des Sauerstoffs eine Selektivitätszunahme hinsichtlich des Zielproduktes Ethylen zur Folge hat [19]. Somit sollte eine Verteilung des Sauerstoffs durch eine Dosierung über eine Membran entlang des Katalysatorbettes in Verbindung mit einer Zuleitung des Eduktes Ethan am Reaktoreintritt die Ethylenselektivität gegenüber dem FBR steigern.

Der Einfluss der unterschiedlichen Zuleitung des Sauerstoffs bzw. der den Sauerstoff enthaltenen Luft in die Reaktionszone wurde mit den in Abb. 3 dargestellten Reaktorkonfigurationen analysiert. Neben dem konventionellen FBR mit einer Zufuhr aller Edukte am Reaktoreintritt wurde die Luft im einstufigen MR entlang des im Inneren der verwendeten porösen $\gamma-\mathrm{Al}_{2} \mathrm{O}_{3}-$ Membran [20] positionierten Katalysators gleichmäßig verteilt. Die vollständige Verteilung der in den Außenraum des MR geströmten Luft entlang des Katalysatorbettes wurde durch eine spezielle Konstruktion des Außenvolumens mit lediglich einem Lufteintritt erreicht.

\section{Abbildung 3.}

Schematische Darstellung der Reaktorkonfigurationen (FBR und MR)

FBR:

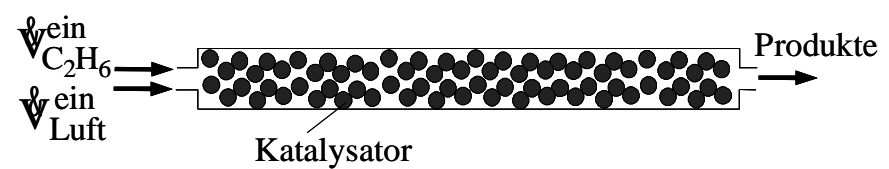

MR:

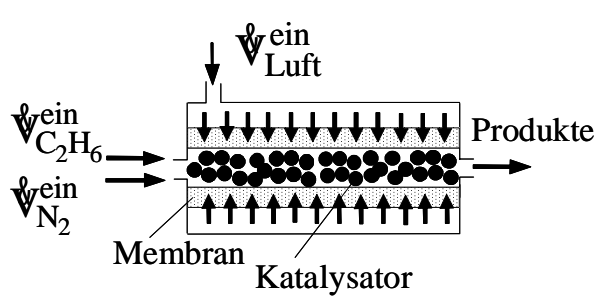


In Abb. 4 sind der Umsatz von Ethan, die integrale Ethylenselektivität und die Ethylenausbeute in Abhängigkeit von der Temperatur sowie die integrale Ethylenselektivität über dem Ethanumsatz bei einem nahezu stöchiometrischen Einsatzverhältnis von Ethan zu Sauerstoff zur Bildung von Ethylen und Wasser (Gleichung 25) für den MR und den konventionellen FBR dargestellt. Die Eintrittsmolanteile von Ethan und Sauerstoff, $x_{O 2}^{\text {ein }}$ und $x_{C 2 H 6}^{e i n}$, bezeichnen die bei Mischung aller dem Reaktor zugeleiteten Stoffströme sich einstellenden Molanteile und wurden wie folgt berechnet:

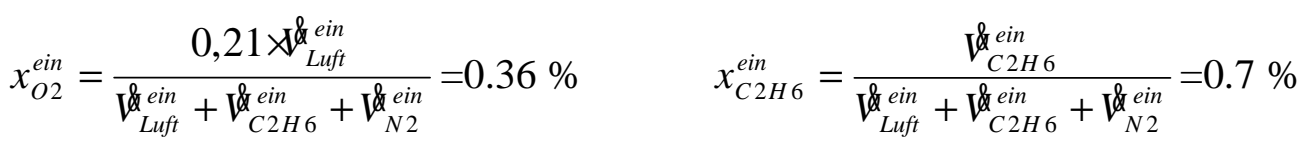

Auf Grund der Luftzugabe über die Membran (Luftmenge entsprach ca. $90 \%$ des Gesamtvolumenstromes) war die mittlere Verweilzeit des Ethans im MR deutlich höher als im FBR. Trotz der mit der Dosierung von mehr als $90 \%$ des Gesamtstromes über die Membran verbundenen höheren Verweilzeit im MR traten infolge der Konzentrationsabsenkung des verteilten Sauerstoffs zunächst bei niedrigen Temperaturen höhere Ethanumsätze im FBR auf (Abb. 4a). Nach dem Schneiden beider Kurven bei ca. 570 ${ }^{\circ} \mathrm{C}$ wurden im MR bei weiterer Temperaturerhöhung höhere Ethanumsätze erzielt.

Abbildung 4.

a) Ethanumsatz, b) Ethylenselektivität und c) Ethylenausbeute in Abhängigkeit von der Temperatur sowie d) Ethylenselektivität über Ethanumsatz für den MR und den FBR bei $\mathrm{O}_{2}$ Unterschuss $\left(x_{O 2}^{\text {ein }}=\mathbf{0 . 3 6} \%, x_{C 2 H 6}^{\text {ein }}=\mathbf{0 . 7} \%, p=1 \mathrm{bar}, \mathrm{GHSV}=\mathbf{1} / \boldsymbol{\tau}=\mathbf{6 0 0 0} \mathrm{h}^{-1}\right)$.
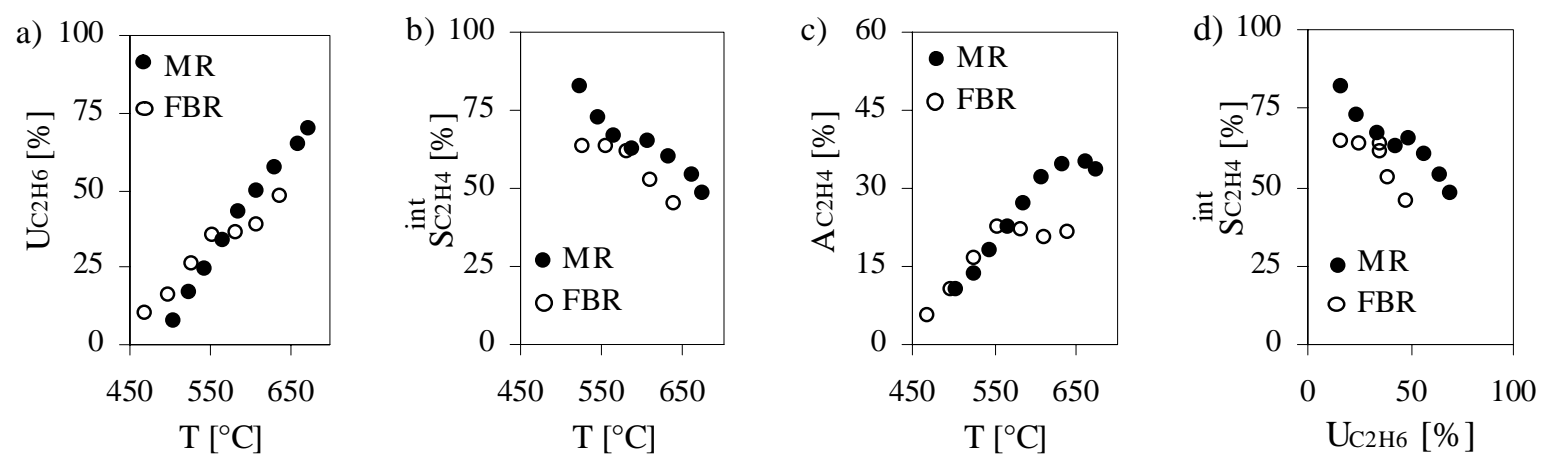

Aus Abb. 4b wird deutlich, dass die Verteilung des in der Luft enthaltenden Sauerstoffs im MR im gesamten Temperaturbereich eine höhere Ethylenselektivität als im FBR zur Folge hatte. Die Erhöhung der Selektivität wurde durch die Absenkung der Sauerstoffkonzentration infolge der Verteilung im MR verursacht. Mit den bei Temperaturen über $600{ }^{\circ} \mathrm{C}$ höheren 
Umsätzen und Selektivitäten im MR ließen sich beachtliche Steigerungen der Ethylenausbeute (Abb. 4c) und damit eine merkliche Verbesserung der Selektivität-UmsatzCharakteristik (Abb. 4d) im Vergleich zum FBR erzielen.

\section{Zusammenfassung}

Für Folge- und Parallelreaktionen wurde demonstriert, dass in Rohrreaktoren unter isothermen Bedingungen der Austrittsmolanteil bzw. die Ausbeute eines Zielproduktes durch eine optimale Verteilung eines Eduktes gesteigert werden kann. Es wurde gezeigt, dass zur Maximierung der Ausbeute die differentielle Selektivität an jeder Reaktorposition optimal eingestellt werden muss. In Abhängigkeit von den konkreten reaktionskinetischen Ansätzen ergibt sich damit ein optimaler, verteilt in die Reaktionszone zu dosierender Strom.

Am Beispiel der oxidativen Dehydrierung von Ethan zu Ethylen konnte mit Hilfe eines einstufigen Membranreaktors mit einer Zugabe von Ethan am Reaktoreintritt (verdünnt mit Stickstoff) und einer Zugabe der den Sauerstoff enthaltenen Luft über die durchlässige Reaktorwand experimentell demonstriert werden, dass durch eine Absenkung der Sauerstoffkonzentration im Reaktionsraum infolge der $\mathrm{O}_{2}$-Verteilung die Ethylenselektivität im Membranreaktor gegenüber dem Festbettreaktor deutlich gesteigert werden kann. Gleichzeitig konnten eine Verbesserung der Selektivität-Umsatz-Charakteristik und damit eine Ausbeuteerhöhung des Zielproduktes Ethylen erzielt werden.

Die Autoren danken der Deutschen Forschungsgemeinschaft (DFG) für die finanzielle Unterstützung im Rahmen der an der Otto-von-Guericke-Universität Magdeburg eingerichteten DFG-Forschergruppe „Membranunterstützte Reaktionsführung“. Weiterhin danken wir dem Hermsdorfer Institut für Technische Keramik (HITK) für die im Rahmen dieses Projektes eingesetzten Membranen und Herrn M.Sc. Jitendra Kumar für den Hinweis auf das Leibnitz Integral.

\section{Formelzeichen}

$\begin{array}{lll}A_{i} & {[\%]} & \text { Ausbeute der Komponente } i \\ D_{\text {Rohr }} & {[\mathrm{m}]} & \text { Rohrreaktordurchmesser } \\ E & {[-]} & \text { Eduktkomponente } \\ F & {\left[\mathrm{~m}^{2}\right]} & \text { Reaktorquerschnittsfläche } \\ G P & {[-]} & \text { gewünschtes Produkt } \\ G R & {[-]} & \text { gewünschte Reaktion } \\ i & {[-]} & \text { Komponente } i\end{array}$




$\begin{array}{ll}j & {[-]} \\ k_{j} & {\left[\mathrm{~mol} \cdot \mathrm{s}^{-1} \cdot \mathrm{m}^{-3}\right]} \\ M & {[-]} \\ \underset{\underbrace{\text { gin }}_{\text {ges }}}{ } & {[\mathrm{mol} / \mathrm{s}]} \\ r_{j} & {\left[\mathrm{~mol} \cdot \mathrm{s}^{-1} \cdot \mathrm{m}^{-3}\right]} \\ S_{i}^{\text {diff }} & {[\%]} \\ S_{i}^{\text {int }} & {[\%]} \\ T & {[\mathrm{~K}]} \\ U_{i} & {[\%]} \\ U P & {[-]} \\ U R & {[-]} \\ x_{i} & {[-]} \\ & \\ \alpha & {[-]} \\ v_{i j} & {[-]} \\ \xi & {[-]} \\ \tau & {[\mathrm{s}]}\end{array}$

Reaktion $j$

Geschwindigkeitskonstante der Reaktion $j$

Anzahl der Reaktionen

Gesamtmolenstrom am Reaktoreintritt

Geschwindigkeit der Reaktion $\mathrm{j}$

differentielle Selektivität hinsichtlich der Komponente $i$

integrale Selektivität hinsichtlich der Komponente $i$

Temperatur

Umsatz des Eduktes i

unerwünschtes Produkt

unerwünschte Reaktion

Molanteil der Komponente i

\section{Literatur}

[1] G. Ertl, H. Knözinger, J. Weitkamp, Handbook of Heterogeneous Catalysis, Vol. 1-5, Wiley-VCH, Weinheim 1997.

[2] B. Cornils, W. A. Herrmann, R. Schlögl, C.-H. Wong, Catalysis from A to Z, A Concise Encyclopedia, Wiley-VCH GmbH \& Co. KgaA, 2003.

[3] R.A. Sheldon, R.A. van Santen, Catalytic Oxidation, World Scientific, 1995.

[4] B.K. Hodnett, Heterogeneous Catalytic Oxidation: Fundamental and Technological Aspects of the Selective and Total Oxidation of Organic Compounds, Wiley, 2000.

[5] K.R. Westerterp, W.P.M. van Swaaij, A.A.C.M. Beenackers, Chemical Reactor design and operation, John Wiley \& Sons, 1984.

[6] T.F. Edgar, D.M. Himmelblau, Optimization of Chemical Processes, McGraw-Hill, 1988.

[7] M. Morbidelli, A. Gavriilidis, A. Varma, Catalyst Design: Optimal Distribution of Catalyst in Pellets, Reactors and Membranes, Cambridge University Press, Cambridge 2001.

[8] O. Levenspiel, Chemical Reaction Engineering, John Wiley \& Sons, 1972.

[9] Y.L. Lu, A.G. Dixon, W.R. Moder, Y.H. Ma, Chem. Eng. Sci. 1997, 52, 1349.

[10] Y.L. Lu, A.G. Dixon, W.R. Moder, Y.H. Ma, Catalysis Today 1997, 35, 443.

[11] J.G. Sanchez Marcano, T.T. Tsotsis, Catalytic Membranes and Membrane Reactors, Wiley-VCH, 2002.

[12] S. Thomas, Dissertation, Fakultät für Verfahrens- und Systemtechnik, Otto-vonGuericke-Universität Magdeburg 2003.

[13] M. Baerns, H. Hofmann, A. Renken, Chemische Reaktionstechnik, Georg Thieme Verlag, Stuttgart, 1999.

[14] John R. Fanchi, Math refresher for scientists and engineers, John Wiley \& Sons, New York, 1997.

[15] C. Hamel, S. Thomas, K. Schädlich, A. Seidel-Morgenstern, Chem. Eng. Sci. 2003, 58, 4483. 
[16] S. Thomas, S. Pushpavanam, A. Seidel-Morgenstern, Ind. Eng. Chem. Res. 2004, 43, 969.

[17] W. Press, B. Flannery, S. Teukolsky, W.T. Vetterling, Numerical Recipes, Cambridge University Press, 1992.

[18] F. Klose, T. Wolff, S. Thomas, A. Seidel-Morgenstern, Applied Catal. A: General, 2004, 257, 193.

[19] F. Klose, T. Wolff, S. Thomas, A. Seidel-Morgenstern, Catal. Today 2003, 82 , 25.

[20] S. Thomas, R. Schäfer, J. Caro, A. Seidel-Morgenstern, Catal. Today 2001, 67, 205. 